\title{
Aortic arch calcification is strongly associated with obstructive sleep apnea
}

\author{
(1)Çağrı Zorlu \\ Tokat Gaziosmanpaşa University, Department of Cardiology, Tokat, Turkey
}

Cite this article as: Zorlu Ç. Aortic arch calcification is strongly associated with obstructive sleep apnea. J Health Sci Med 2021; 4(6): 779-783.

\begin{abstract}
Aim: Obstructive sleep apnea (OSA) is a common clinical condition that causes an increase in cardiovascular morbidity and mortality. OSA is likely to show increased arterial stiffness and progressive systemic atherosclerosis. Chest radiography reveals atherosclerotic changes in the aorta.The aim of this study was to investigate the relationship between aortic arch calcification (AAC) on chest radiography and OSA.

Material and Method: 204 patients (age: 55 \pm 14 years; 78 men) who were diagnosed with OSA by performing night polysomnography were evaluated. On the other hand 200 (age: $48 \pm 15$ years; 94 men) patients were selected to the group non OSA. AAC was evaluated with chest radiography and inter-observer agreement was analyzed by using kappa statistics. Univariate and multivariate logistic regression analysis was conducted to assess the association of AAC and OSA. P-value $<0.05$ was considered statistically significant.

Result: The prevalence of AAC was 207 (51,2\%). OSA group had significantly higher prevalence of AAC (79\% vs. 32.5\%, p $<0.0001$ ) as compared to the normal group. Presence of AAC was a strong and independent predictor of OSA (OR 3.923, $95 \%$ CI 2.396 to 6.328 ) in multivariate analysis.

Conclusion: Presence of AAC on plain chest radiography is strongly and independently associated with the presence of OSA.
\end{abstract} Keywords: Aorta, thoracic, calcinosis, sleep apnea, obstructive.

\section{INTRODUCTION}

Obstructive sleep apnea (OSA) is a disease that causes complete or partial airway collapse during sleep and thus repetitive ventilation interruptions (1). OSA has been demonstrated to be involved with increased prevalence of cardiovascular disease (CVD) due to intermittent sleep interruptions, intermittent chronic hypoxia, and changes in intra-chest pressure $(2,3)$. Oxidative stress and sympathetic activation occur in OSA due to fast reoxygenation following periods of hypoxia. These also lead to inflammation resulting in endothelial dysfunction and thus subclinical and clinical atherosclerosis (4).

In some studies, the relationship between OSA and coronary artery calcium level has been revealed and, as it is known, coronary artery calcium level is one of the indicators of subclinical atherosclerosis (5). There are previous studies evaluating the relationship between aortic calcification, coronary artery calcium level, and CVD (6). Calcium accumulation in the aortic arch can be evaluated by chest radiography. Calcification of the aortic arch gives preliminary clues about atherosclerosis. Based on all these, further investigation of the relationship between OSA and subclinical atherosclerosis markers is valuable in that it leads to evaluations for reducing possible future adverse events related to CVD in patients with OSA.

In this study, it was aimed to research its relationship with OSA by evaluating aortic arch calcification (AAC) on chest radiography.

\section{MATERIAL AND METHOD}

The study was carried out with the permission of Tokat Gaziosmanpaşa University Faculty of Medicine Clinical Researchs Ethics Committee (Date: 19.08.2020, Decision No: 20-KAEK-122). All procedures were carried out in accordance with the ethical rules and the principles of the Declaration of Helsinki. 


\section{Study Populations}

The aortic arch was evaluated with posterior-anterior (PA) chest radiography of all participants included in the study. Patients older than 18 years of age and had night polysomnography from July 2018 to May 2020 were included in the study. The conditions for not being included in the study were as follows: inadequate chest $\mathrm{x}$-ray, known aortic, coronary artery, cerebrovascular and peripheral vascular disease, open chest surgery, heart failure, moderate to severe cardiac valve disease, pregnancy, malignancy, and receiving dialysis. All participants were evaluated by transthoracic echocardiography. Participants were evaluated with medical history, physical examination and laboratory data.

\section{Demographic and Laboratory Data}

Biochemical parameters were automatically evaluated with the aid of the Beckman Coulter LH-750 Hematology Analyzer (Beckman Coulter, Inc, Fullerton, California). Routine biochemical parameters were evaluated by standard methods. Hypercholesterolemia was described as an initial cholesterol level of $>200 \mathrm{mg} / \mathrm{dL}$ and/or a low-density lipoprotein cholesterol level of $>130 \mathrm{mg} / \mathrm{dL}$ or pre-diagnosed and treated hypercholesterolaemia. Diabetes mellitus and hypertension were defined as drug users for the treatment of these diseases. In addition to these, presence of diabetes mellitus was determined based on when glucose level was $\geq 126 \mathrm{mg} / \mathrm{dL}$ in several measurements for fasting or glucose level $>200 \mathrm{mg} / \mathrm{dL}$ at any measurement and hypertension was diagnosed based on when repeated systolic/diastolic blood pressure measurements was $\geq 140 / 90 \mathrm{mmHg}$. Current smoking was defined as smoking in the previous 6 months. Body mass index was expressed as $\mathrm{kg} / \mathrm{m}^{2}$.

\section{Evaluation of Obstructive Sleep Apnea}

All subjects were evaluated using polysomnography overnight using an equipped digital polygraph (SomnoStar Alpha Sleep System, SensorMedics Corp., Yorba Linda, CA, USA). Patients who were diagnosed with OSA according to the accepted definition and scoring methods were included in the study (7). Hypopneas were defined as a $30-80 \%$ reduction in nasal pressure flow for at least 10 seconds with oxygen desaturation $\geq 4 \%$. A flow reduction of $80 \%$ in the nasal pressure signal for at least 10 seconds was defined as apneas. Oxygen desaturation scored if there is a $\geq 4 \%$ decrease. The apnea-hypopnea index (AHI) was defined as the number of apnea and hypopneas per hour of the analyzed recording time and it was used to determine the risk of OSA.

\section{The Aortic Arch Assessment}

To all participants included in the study; standard PA chest radiography was taken while the participants were standing (AXIOM Aristos MX, SIEMENS, Germany). The AAC was evaluated in four categories: grade 0 , no visible calcification; grade 1 , the calcification appears as a small dot or thin line; grade 2, calcification at one or more points or in the form of a thick line; grade 3 , circular prominent calcification of the aortic arch (8). AAC for each patient was assessed by two independent, experienced cardiologists in a blinded fashion.

\section{Echocardiographic Examination}

All echocardiographic evaluations (General Electric Vivid S5, Milwaukee, WI, USA) were performed on all participants in the left decubitus position using a 2.5-3.5 $\mathrm{MHz}$ transducer. The examination was performed using two-dimensional and pulsed Doppler measurements according to the American Echocardiography Association and the European Society of Cardiovascular Imaging criteria. Simpson's method was used to evaluate left ventricular ejection fraction (9).

\section{Statistical Analysis}

Statistical analyses were carried out using the SPSS 18.0 Statistical Package Program for Windows (SPSS Inc., Chicago Illinois, USA). Qulitative data are presented as medians with interquartile ranges. Quantitative variables were expressed as a mean value standard deviation and categorical variables as percentages. The distribution of continuous variables was determined with the Kolmogorov-Smirnov test. Student t-test was used to evaluate normally distributed data and MannWhitney $U$ test was used to evaluate non-normally distributed data. Fisher's exact or chi-square tests were used to evaluate categorical variables . Multivariate logistic regression analyses were applied to determine independent factors. Data from univariate and multivariate logistic regression analyses were reported as odds ratios with $95 \%$ CI. A p-value $<0.05$ was considered statistically significant.

\section{RESULTS}

A total of 404 participitians were included in this study. The mean patient age was $55 \pm 14$ years, and 172 (42.5\%) of the patients were men. Two hundred four patients (50.4\%) with OSA were evaluated as an OSA (+) group. Those without OSA diagnosis, symptoms or signs were determined as the OSA (-) group. As compared to the OSA (+) group, the OSA (-) group was older $(\mathrm{p}<0.001)$. Basic characteristic features of the groups, demographic and laboratory data were similar except age. Patient characteristics are summarized in Table $\mathbf{1}$.

In the evaluations made on chest radiography, the frequency of AAC in the group with OSA $(+)$ was statistically higher than the group with OSA $(-)(\mathrm{p}<0.001)$

(Table 2). 
In univariate analyses (Table 3), AAC was positively and significantly correlated with age $(\mathrm{r}=0.172, \mathrm{p}<0.001$, BMI $(\mathrm{r}=0.201, \mathrm{p}<0.001)$, AHI $(\mathrm{r}=0.212, \mathrm{p}<0.001)$, arousal index $(\mathrm{r}=0.282, \mathrm{p}<0.01)$, and $3 \%$ ODI $(\mathrm{r}=0.224, \mathrm{p}<0.01)$, as well as several clinical variables.

\begin{tabular}{|lccc|}
\hline \multicolumn{4}{|c|}{ Table 1. Baseline characteristics of the study groups } \\
\hline & $\begin{array}{c}\text { OSA (+) } \\
(\mathbf{n = 2 0 4 )}\end{array}$ & $\begin{array}{c}\text { OSA (-) } \\
(\mathbf{n}=\mathbf{2 0 0})\end{array}$ & $\begin{array}{c}\text { P } \\
\text { value }\end{array}$ \\
\hline Age (years) & $55 \pm 14$ & $48 \pm 15$ & $<0.001$ \\
Male, $\mathrm{n}(\%)$ & $78(38.2)$ & $94(47)$ & 0.064 \\
BMI, kg/m2 & $29 \pm 5$ & $28 \pm 4$ & 0.062 \\
Smoker, $\mathrm{n}(\%)$ & $65(31.8)$ & $52(26)$ & 0.110 \\
Hypertension, n (\%) & $104(50.9)$ & $83(41.5)$ & 0.051 \\
Glucose (mg/dL) & $106 \pm 30$ & $102 \pm 29$ & 0.086 \\
Diabetes mellitus, n (\%) & $46(22.5)$ & $34(17)$ & 0.286 \\
Total cholesterol (mg/dL) & $189 \pm 40.6$ & $194 \pm 40.4$ & 0.198 \\
Triglyceride (mg/dL) & $191 \pm 39$ & $174 \pm 81$ & 0.019 \\
Low-density lipoprotein (mg/dL) & $116 \pm 38$ & $118 \pm 37$ & 0.902 \\
High-density lipoprotein (mg/dL) & $46 \pm 12$ & $45 \pm 13$ & 0.498 \\
Creatinine (mg/dL) & $0.82 \pm 0.18$ & $0.82 \pm 0.27$ & 0.880 \\
Antihypertensive drug, n & 47 & 35 & 0.049 \\
Antidiabetic drug, n & 2 & 1 & 0.988 \\
AHI, /h & $50.5 \pm 14.4$ & $2.1 \pm 1.7$ & $<0.001$ \\
Aurosal index, /h & $48 \pm 17.2$ & $19.8 \pm 9.6$ & $<0.001$ \\
ODI (3\%), /h & $40.1 \pm 20.2$ & $0.8 \pm 0.7$ & $<0.001$ \\
Lowest SpO2, \% & $75 \pm 9.7$ & $90.5 \pm 3.5$ & $<0.001$ \\
\hline $\begin{array}{l}\text { AHI, apnea-hypopnea index; BMI, body mass index; ODI, oxygen desaturation index; } \\
\text { SpO2, peripheral oxygen saturation }\end{array}$ & & & \\
\hline
\end{tabular}

\begin{tabular}{|lccc|}
\hline \multicolumn{4}{|l|}{ Table 2. Aortic arch calcification grades in the study groups } \\
\hline $\begin{array}{l}\text { Aortic arch } \\
\text { calcification (n, \%) }\end{array}$ & $\begin{array}{l}\text { OSA (+) } \\
(\mathbf{n = 2 0 4})\end{array}$ & $\begin{array}{l}\text { OSA (-) } \\
(\mathbf{n}=\mathbf{2 0 0 )}\end{array}$ & P value \\
\hline Grade 0 & $62(30.3)$ & $135(67.5)$ & $<0.0001$ \\
Grade 1 & $86(42.1)$ & $50(25)$ & $<0.001$ \\
Grade 2 & $45(22)$ & $14(7)$ & $<0.001$ \\
Grade 3 & $10(4.9)$ & $1(0.5)$ & $<0.001$ \\
\hline OSA, obstructive sleep apnea & & & \\
\hline
\end{tabular}

Table 3. Univariate analysis for obstructive sleep apnea

\begin{tabular}{|lcc|}
\hline Variables & $\boldsymbol{\beta}$ & P value \\
\hline Age & 0.172 & $<0.001$ \\
Male & 0.006 & 0.056 \\
BMI & 0.201 & $<0.001$ \\
Smoker & -0.186 & 0.103 \\
Hypertension (\%) & 0.526 & 0.048 \\
Diabetes mellitus (\%) & 0.438 & 0.301 \\
Total cholesterol (mg/dL) & -0.012 & 0.235 \\
Triglyceride (mg/dL) & -0.002 & 0.089 \\
Antihypertensive drug & 0.490 & 0.044 \\
Antidiabetic drug & 0.042 & 0.382 \\
AHI & 0.212 & $<0.001$ \\
Aruosal index & 0.282 & $<0.001$ \\
ODI (\%3) & 0.224 & $<0.001$ \\
Lowest SpO2 & -0.134 & 0.001 \\
\hline AHI, apnea-hypopnea index; BMI, body mass index; ODI, oxygen desaturation index; \\
SpO2, periphral oxyen saturation
\end{tabular}

ACC was associated with OSA in univariate logistic regression analysis (Table 4). According to the results of multivariate regression analysis, AAC was also identified as an independent predictor for OSA ( $\mathrm{r}=1.362$, OR 3.923, 95\%CI 2.396 to 6.328) (Table 5).

\begin{tabular}{|lcc|}
\hline \multicolumn{3}{|l|}{ Table 4. Correlations between OSA and AAC } \\
\hline Aortic arch calcification (\%) & $\boldsymbol{\beta}$ & P value \\
\hline Grade 0 & Reference category \\
Grade 1 & 1.384 & $<0.0001$ \\
Grade 2 & 1.846 & $<0.0001$ \\
Grade 3 & 2.698 & 0.006 \\
\hline ACC, aortic arch calcification; OSA, obstructive sleep apnea & \\
\hline
\end{tabular}

\begin{tabular}{|lcccc|}
\hline \multicolumn{5}{|l}{ Table 5. Multivariate analysis for obstructive sleep apnea } \\
\hline Variables & $\boldsymbol{\beta}$ & OR & Lower & Upper \\
\hline Age & 0.014 & 1.014 & 0.978 & 1.034 \\
Body mass index & 0.035 & 1.036 & 0.987 & 1.089 \\
Hypertension & 0.060 & 1.062 & 0.658 & 1.690 \\
Triglyceride & -0.002 & 0.985 & 0.989 & 0.996 \\
$\begin{array}{l}\text { Presence of aortic } \\
\text { arch calcification }\end{array}$ & 1.362 & 3.923 & 2.396 & 6.328 \\
AHI & 0.15 & 1.018 & 0.985 & 1.049 \\
Male & 0.15 & 1.008 & 0.964 & 1.027 \\
\hline AHI, apnea-hypopnea index & & & & \\
\hline
\end{tabular}

\section{DISCUSSION}

As far as we know, this study clearly reveals the relationship between AAC and OSA, which was detected for the first time via chest radiography. Therefore, the detection of ACC, which is one of the indicators of subclinical atherosclerosis, more frequently in OSA patients provides early treatment and approaches. Accordingly, this situation; it is important in terms of improving the morbidity and mortality of CVD.

The pathophysiology of OSA is not fully explained. Factors that reduce the width of the upper respiratory tract can cause OSA (10). The most important complications seen in patients with OSA are associated to the cardiovascular system (11). Cardiovascular diseases seen with OSA in order of frequency: hypertension (30-60\%), CAD (20$30 \%)$, pulmonary hypertension $(20-30 \%)(12,13)$.

Many risk factors for atherosclerosis are common with OSA; age, male gender, smoking, obesity, metabolic syndrome are parameters specific to both situations (14). Oxidative stress in OSA; there are data that it causes both endothelial dysfunction and LDL oxidation (15). CRP, fibrinogen, IL- 6 are risk factors for atherosclerosis and these have also been shown to increase in OSA (16). In patients with CAD proven by coronary angiography, OSA has been found frequently (17).

Local endothelial damage, inflammation, oxidative stress, and vascular calcification are involved in the pathogenesis of atherosclerosis (18). Calcification of the 
arterial wall is part of atherosclerosis and is not found in normal vessels (19).

Oxidative stress and inflammatory reaction in OSA lead to increased arterial stiffness, carotid intimamedia thickness and CAD progression (20-22). OSA is frequently seen in patients with aortic aneurysms and dissection and it has been suggested that negative intrathoracic pressure may play a role in the development or worsening of these pathologies. Additionally, negative intrathoracic pressure is thought to cause basal atherosclerotic changes in the aorta $(23,24)$.

AAC has been presented as a substitution marker for atherosclerosis to better reflect the total burden of atherosclerosis (25). Atherosclerosis may be suspected if ACC is present in the chest radiography evaluation in OSA patients, and so patients can be stratified by their risk of atherosclerosis (26). In addition, Patients with higher risk for atherosclerosis can be distinguished by investigating the presence of ACC in patients with OSA. Most of the parameters that are indicators of subclinical atherosclerosis do not provide additional information other than the prediction of subclinical atherosclerosis (27-29). On the other hand, chest radiography is generally used in OSA, health checks unrelated to OSA, and in various clinical settings.

In this study, we have demonstrated that the presence of AAC is strongly associated with OSA. New studies are also needed to confirm our findings and, in addition, to assess the possible relationship of AAC and OSA by classifying by severity.

\section{Study Limitations}

The main limitation of the study is the small number of participants included in the study. OSA is categorized as no/mild, moderate, and severe. However, we did not examine OSA by classifying it by severity.

\section{CONCLUSION}

OUA is strongly and independently associated with ACC presence on plain chest radiography. This simple assessment allows us to distinguish patients with OSA based on their risk of developing subclinical atherosclerosis.

\section{ETHICAL DECLARATIONS}

Ethics Committee Approval: The study was carried out with the permission of Tokat Gaziosmanpaşa University Faculty of Medicine Clinical Research Ethics Committee (Date: 19.08.2020, Decision No: 20-KAEK-122).

Informed Consent: Because the study was designed retrospectively, no written informed consent form was obtained from patients.
Referee Evaluation Process: Externally peer-reviewed. Conflict of Interest Statement: The authors have no conflicts of interest to declare.

Financial Disclosure: The authors declared that this study has received no financial support.

Author Contributions: All of the authors declare that they have all participated in the design, execution, and analysis of the paper, and that they have approved the final version.

Congress Information: The abstract text was presented at the 36th Turkish Cardiology Congress with international participation at 03.12.2020.

\section{REFERENCES}

1. Somers VK, White DP, Amin R. Sleep apnea and cardiovascular disease. Circulation 2008; 118: 1080-111.

2. Kepez A, Niksarlığlu EY, Hazırolan T, et al. Evaluation of association between obstructive sleep apnea and coronary risk scores predicted by tomographic coronary calcium scoring in asymptomatic patients. Anadolu Kardiyol Derg 2011: 428-35.

3. Kasai T, Floras JS, Bradley TD. Sleep apnea and cardiovascular disease: A bidirectional relationship. Circulation 2012; 126: 1495510.

4. Korkmaz L, Adar A, Korkmaz A, et al. Aortic knob calcification and coronary artery lesion complexity in non-ST-segment elevation acute coronary syndrome patients. Turk Kardiyol Dern Ars 2012; 40: 606-11.

5. Sorajja D, Gami AS, Somers VK. Independent association between obstructive sleep apnea and subclinical coronary artery disease. Chest 2008; 133: 927-33.

6. Pressman GS, Crudu V, Parameswaran-Chandrika A, RomeroCorral A, Purushottam B, Figueredo VM. Can total cardiac calcium predict the coronary calcium score? Int J Cardiol 2011; 146: 202-6.

7. Levey AS, Stevens LA, Schmid CH, et al. A new equation to estimate glomerular filtration rate. Ann Intern Med 2009; 150: 604-12.

8. Silber $\mathrm{MH}$, Ancoli-Israel S, Bonnet $\mathrm{MH}$, et al. The visual scoring of sleep in adults. J Clin Sleep Med 2007; 3: 121-31.

9. Symeonidis G, Papanas N, Giannakis I, et al. Gravity of aortic arch calcification as evaluated in adult Greek patients. Int Angiol 2002; 21: 233-6.

10.Sahn D.J, Maris A, Kisslo J. For the committee on M-mode standardization of the American Society of Echocardiography Recommendation regarding quantitation in M-mode echocardiographic measurements. Circulation 1978; 58: 1072-83.

11.Strauss RS, Browner WS. Risk for obstructive sleep apnea. Ann Intern Med 2000; 132: 758-9.

12. Hedner J, Grote L. Cardiovascular consequences of obstructive sleep apnea. Eur Respir Mon 1998; 10: 227-65.

13. Phillips B. Sleep-disordered breathing and cardiovascula rdisease. Sleep Med Rev 2005; 9: 131-40.

14. Paris JM, Somers VK. Obstructive sleep apnea and cardiovascular disease. Mayo Clin Proc 2004; 79: 1036-46.

15. Yokoe T, Minoguchi K, Matsuo H. Elevated levels of C-reactive protein and interleukin-6 in patients with obstructive sleep apnea syndrome are decreased by nasal continuous positive air way pressure. Circulation 2003;107: 1129- 33.

16. Schäfer H, Koehler U, Ploch T, Peter JH. Sleep-related myocardial ischemia and sleep structure in patients with obstructive sleep apnea and coronary heart disease. Chest 1997; 111: 387-93. 
17. Punjabi NM, Sorkin JD, Katzel LI, Goldberg AP, Schwartz AR, Smith PL. Sleep-disordered breathing and insulin resistance in middle-aged and overweight men. Am J Respir Crit Care Med 2002; 165: 677-82.

18. Coughlin SR, Mawdsley L, Mugarza JA. Obstructive sleep apnea is indepently associated with an increased prevalence of metabolic syndrome Eur Heart J 2004; 25: 735-41.

19. Mintz GS, Popma JJ, Pichard AD, et al. Patterns of calcification in coronary artery disease. A statistical analysis of intravascular ultrasound and coronary angiography in 1155 lesions. Circulation. 1995; 91: 1959-65.

20.Stary HC, Chandler AB, Dinsmore RE, et al. A definition of advanced types of atherosclerotic lesions and a histological classification of atherosclerosis. A report from the Committee on Vascular Lesions of the Council on Arteriosclerosis, American Heart Association. Arterioscler Thromb Vasc Biol 1995; 15: 151231

21. Kinoshita T, Yahaba M, Terada J, et al. Impact of arterial stiffness on WatchPAT variables in patients with obstructive sleep apnea. J Clin Sleep Med 2018; 14: 319-25.

22. Drager LF, Bortolotto LA, Figueiredo AC, Krieger EM, Lorenzi GF. Effects of continuous positive airway pressure on early signs of atherosclerosis in obstructive sleep apnea. Am J Respir Crit CareMed 2007; 176: 706-12.

23. Shpilsky D, Erqou S, Patel SR, et al. Association of obstructive sleep apnea with microvascular endothelial dysfunction and subclinical coronary artery disease in a community-based population. Vasc Med 2018; 23: 331-9.

24. Mason RH, Ruegg G, Perkins J, et al. Obstructive sleep apnea in patients with abdominal aortic aneurysms: highly prevalent and associated with aneurysm expansion. Am J Respir Crit Care Med 2011; 183: 668-74.

25. Korkmaz L, Erkan H, Korkmaz AA, et al. Relationship of aortic knob width with cardio-ankle vascular stiffness index and its value in diagnosis of subclinical atherosclerosis in hypertensive patients: a study on diagnostic accuracy. Anadolu Kardiyol Derg 2012; 12: 102-6.

26. Bos D, Leening MJ, Kavousi M, et al. Comparison of atherosclerotic calcification in major vessel beds on the risk of all-cause and cause-specific mortality: The Rotterdam study. Circ Cardiovasc Imaging 2015; 8: e003843.

27. Tomita Y, Kasai T, Ishiwata S, Daida H, Narui K. Aortic knob width as a novel indicator of atherosclerosis and obstructive sleep apnea. J Atheroscler Thromb 2020; 27: 501-8.

28.Zylla S, Dörr M, Völzke $H$, et al. Association of circulating chemerin with subclinical parameters of atherosclerosis: results of a population-based study. Arterioscler Thromb Vasc Biol 2018, 38: 1656-64.

29. Franzago M, Fraticelli F, DiNicola M, et al E. Early subclinical atherosclerosis in gestational diabetes: the predictive role of routine biomarkers and nutrigenetic variants. J Diabetes Res 2018; 2018: 9242579 